Article

\title{
Integrating Spirituality as a Key Component of Patient Care
}

\author{
Suzette Brémault-Phillips ${ }^{1, *}$, Joanne Olson ${ }^{2}$, Pamela Brett-MacLean ${ }^{3,4}$, Doreen Oneschuk ${ }^{5}$, \\ Shane Sinclair ${ }^{6}$, Ralph Magnus ${ }^{7}$, Jeanne Weis ${ }^{8}$, Marjan Abbasi ${ }^{9}$, Jasneet Parmar ${ }^{9}$ \\ and Christina M. Puchalski ${ }^{10,11}$
}

1 Department of Occupational Therapy, Faculty of Rehabilitation Medicine, 2-64 Corbett Hall, University of Alberta, Edmonton AB T6G 2G4, Canada

2 Faculty of Nursing, 4-299 Edmonton Clinic Health Academy (ECHA), University of Alberta, Edmonton AB T6G 1C9, Canada; E-Mail: joanne.olson@ualberta.ca

3 Arts \& Humanities in Health \& Medicine (Undergraduate Medical Education Program), Faculty of Medicine \& Dentistry, 1-001 Katz Group Centre, University of Alberta, Edmonton AB T6G 2E1, Canada; E-Mail: pbrett@ualberta.ca

4 Department of Psychiatry, Faculty of Medicine \& Dentistry, 1E1 Walter Mackenzie Health Sciences Centre, University of Alberta, Edmonton AB T6G 2B7, Canada

5 Division of Palliative Medicine, Department of Oncology, Faculty of Medicine \& Dentistry, University of Alberta, Edmonton AB T6L 5X8, Canada;

E-Mail: doreen.oneschuk@albertahealthservices.ca

6 Faculty of Nursing, University of Calgary, Calgary AB T2N 1N4, Canada; E-Mail: sinclair@ucalgary.ca

7 Spiritual Care, Covenant Health, Edmonton AB T6L 5X8, Canada;

E-Mail: ralph.magnus@covenenthealth.ca

8 Faculty of Health and Community Studies, NorQuest College, Edmonton AB T5J 1L6, Canada; E-Mail:weis@ualberta.ca

9 Department of Family Medicine, Faculty of Medicine \& Dentistry, University of Alberta, Edmonton AB T6G 2C8, Canada; E-Mails: mabbasi@ualberta.ca (M.A.); jasneet.parmar@albertahealthservices.ca (J.P.)

10 The George Washington Institute for Spirituality and Health, School of Medicine and Health Sciences, The George Washington University, Washington, DC 20036, USA;

E-Mail: cpuchals@gwu.edu

11 Division of Geriatrics and Palliative Medicine, School of Medicine and Health Sciences, The George Washington University, Washington, DC 20037, USA

* Author to whom correspondence should be addressed;

E-Mail: suzette.bremault-phillips@ualberta.ca; Tel.: +1-780-492-9503.

Academic Editor: Arndt Büssing

Received: 30 January 2015 / Accepted: 2 April 2015 / Published: 17 April 2015 


\begin{abstract}
Patient care frequently focuses on physical aspects of disease management, with variable attention given to spiritual needs. And yet, patients indicate that spiritual suffering adds to distress associated with illness. Spirituality, broadly defined as that which gives meaning and purpose to a person's life and connectedness to the significant or sacred, often becomes a central issue for patients. Growing evidence demonstrates that spirituality is important in patient care. Yet healthcare professionals (HCPs) do not always feel prepared to engage with patients about spiritual issues. In this project, HCPs attended an educational session focused on using the FICA Spiritual History Tool to integrate spirituality into patient care. Later, they incorporated the tool when caring for patients participating in the study. This research (1) explored the value of including spiritual history taking in clinical practice; (2) identified facilitators and barriers to incorporating spirituality into person-centred care; and (3) determined ways in which HCPs can effectively utilize spiritual history taking. Data were collected using focus groups and chart reviews. Findings indicate positive impacts at organizational, clinical/unit, professional/personal and patient levels when HCPs include spirituality in patient care. Recommendations are offered.
\end{abstract}

Keywords: spirituality; spiritual care; healthcare professionals; spiritual history; patient care; interprofessional

\title{
1. Introduction
}

Spirituality, broadly defined as that which gives meaning and purpose to one's life and connectedness to the significant or sacred [1], is often an imminent and central issue for patients experiencing a serious or chronic medical condition, advancing in the aging process, or at the end of life. Puchalski [2] describes spiritual care as attending to the whole person, including physical, emotional, social, and spiritual dimensions of their experience. Broadly conceived, spiritual care is understood as "the foundation of whole person, patient-centred care" [3]. This component of quality care is heightened during illness, throwing into sharp relief compassionate clinical care as an inherently spiritual intervention. When conceptualized as a distinct domain of care, patients have consistently identified addressing spiritual issues as being among the most important and under-addressed end of life need [4-6]. Just as patient-centred care has been linked to healthcare outcomes [7,8], literature has begun to demonstrate the importance of spirituality to patients when making healthcare decisions [9] and coping with end of life distress [10].

Despite being recognized as a vital component of palliative care by patients and policy makers [11], care providers struggle to find ways to address spirituality in patient care [5,12]. Caring for patients with life limiting conditions has primarily taken a biomedical approach focused on cure orientated outcomes and/or physical symptom management [13]. Numerous studies have reported that the majority of patients facing the end of life, whether their spirituality is expressed through religious or secular means, desire to have their spiritual issues addressed by their healthcare providers $[4,14,15]$. Seriously ill patients have also identified spiritual suffering as contributing to patient distress [16], 
and likewise desire that this be attended to. Addressing spirituality at such significant times in life has been noted to buffer against spiritual pain and distress, which was reported at $44 \%$ of patients in one study [17] and $61 \%$ of patients in a separate study [18].

Evidence demonstrates that attending to this important component of patient care can help HCPs more fully understand their patients. Yet, a variety of organizational and structural, as well as professional and personal factors, have been identified that interfere with the provision of spiritual care. At a professional and personal level, some HCPs do not feel competent to engage patients around spiritual issues. Discussion of religious and spiritual issues is often described as a highly advanced communication skills area. Tools to help facilitate such discussion may be helpful. A recent survey of research priorities in spiritual care identified the evaluation of screening tools to address patient spiritual needs as the number one priority amongst an interprofessional (IP) sample $(n=807)$ of European palliative care providers [19]. HCPs have also noted that knowledge gaps can exist, making it difficult for them to meet patients' spiritual needs [20]. Initiating a conversation regarding emotional and spiritual care can be challenging for some HCPs [21], and concerns that engaging in such conversations will require a substantial time commitment [22] are at times a deterrent. When such conversations occur, however, they are often profoundly meaningful to patients, and informative and rewarding for HCPs [14,23]. Given the increasing awareness of the responsibility of all team members to address spirituality [1], professional programs are increasingly offering courses or integrating spirituality within curricula to support the development of HCPs' competencies in this area.

An Inpatient Spiritual Care Implementation Model [ISCIM] was developed to integrate spiritual history and ongoing spirituality-related discussions, as well as assessment of spiritual distress into routine care and treatment planning by IP team members [1]. One of the spiritual history tools suggested is the FICA Spiritual History Tool (FICA) [24-26], developed by Puchalski and Romer [25] as a practical, simple approach for taking a spiritual history within the context of clinical practice (See Box 1 for the FICA Spiritual History Tool).

The FICA (Faith, Importance, Community, Address) includes questions that help to identify the presence of Faith, belief, or meaning; the Importance of spirituality in relation to a patient's life and healthcare decision-making, their spiritual Community; and explore interventions that may be helpful in Addressing the patient's spiritual needs. It also offers a flexible interview guide to help HCPs engage in conversations and invite patients to share their spiritual beliefs and concerns in relation to their illness experience. Borneman et al. [27] conducted an early assessment of the FICA with cancer patients and found the tool to be helpful in identifying the depth and breadth of spirituality-related issues of patients, as well as opportunities for addressing these concerns. The FICA was helpful in ensuring respectful, patient-centred care, supporting the importance of determining spiritual needs in relation to treatment planning, and enhancing quality of life of patients in clinical settings. Borneman et al. recommended further use of the FICA in other practice settings.

Spiritual history taking and assessment are increasingly important activities in contemporary healthcare practice. The goal of the current project was to improve person-centred care by integrating spirituality as a key component of care in the context of IP healthcare teams. The following assumptions guided the current study: (1) education about use of the FICA by members of IP teams within palliative and geriatric care would increase the willingness of HCPs to engage in discussions about spiritual issues with patients; (2) taking a spiritual history would improve communication between patients and HCPs (as indicated by chart documentation and integration of spiritual issues in treatment planning 
and care, including increased referrals to spiritual care professionals (SCPs)), and enhance quality of care and outcomes for patients.

Box 1. FICA spiritual history tool [24-26].

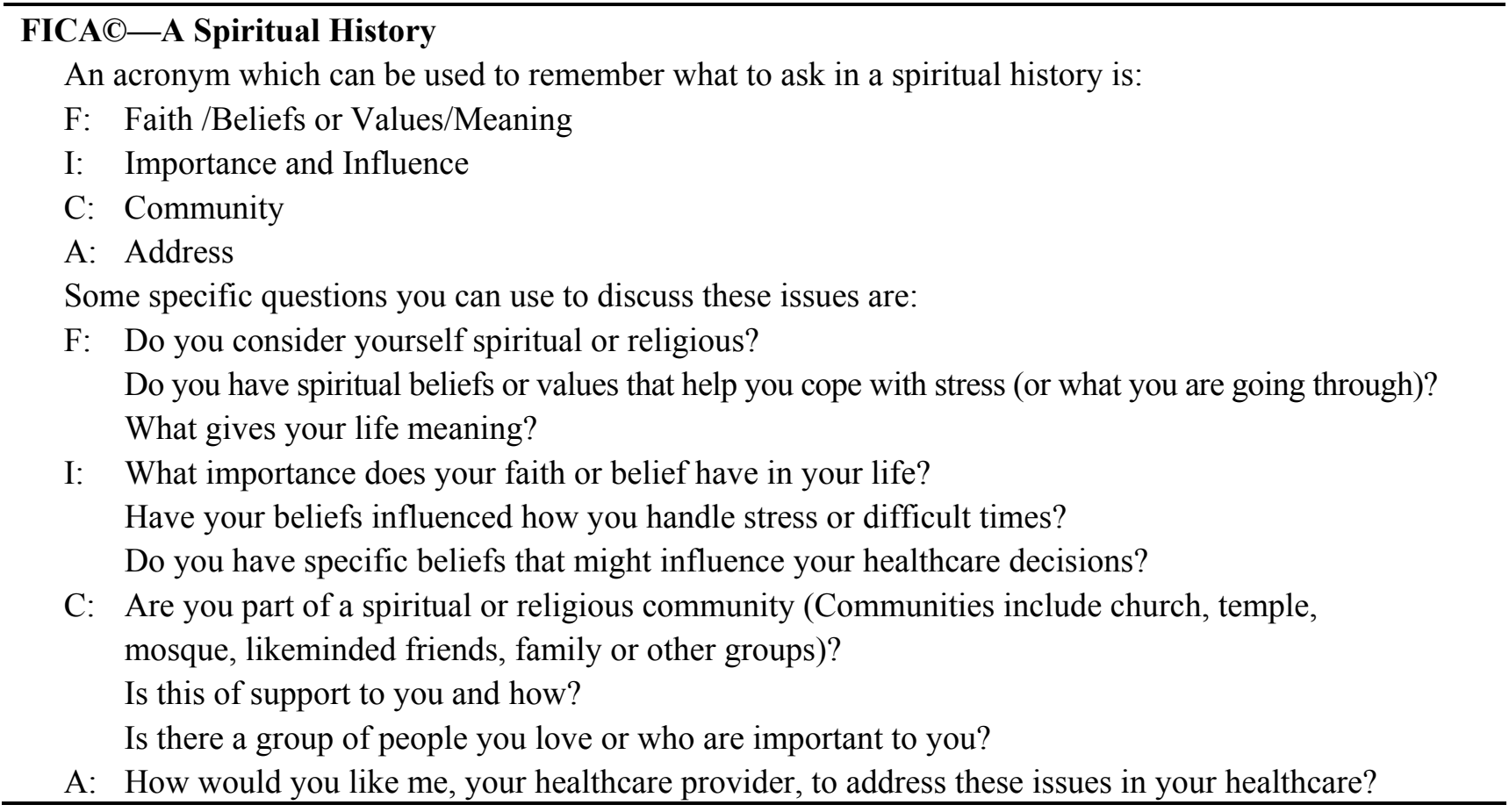

Notes: (C) C. Puchalski, 1996. Please contact Dr. C. Puchalski for permission to use the FICA tool; the "A" can also indicate "Assessment"- spiritual diagnosis, issue or resource of strength and then plan in a treatment/care plan.

\section{Methods}

This descriptive, qualitative exploratory project aimed to examine how use of a spiritual history tool affected provision of patient care. Study objectives were to (1) explore the value of including spirituality in clinical practice; (2) identify facilitators and barriers to incorporating spirituality into person-centred care; and (3) determine ways HCPs can utilize a spiritual history when giving person-centred care. The University of Alberta Health Research Ethics Board approved the study protocol. This pilot intervention generated data, which will inform further proposals for collaborative multisite intervention studies.

\subsection{Setting and Participants}

Three inpatient hospital units in Alberta, Canada operated by Covenant Health (a faith-based health care organization), served as the setting for this research: a hospice unit, a tertiary palliative care unit, and a geriatric assessment unit. The hospice unit is a 24-bed unit serving both advanced cancer patients and patients with life limiting illnesses, who have an estimated prognosis of less than four months. The tertiary palliative care unit is a dedicated 20-bed inpatient unit serving advanced cancer patients and those with life limiting illnesses. A 15-bed inpatient unit, the geriatric assessment unit provides an IP approach to assessment and rehabilitation of frail elderly with complex needs. Patients generally have multiple co-morbidities, some of which would be considered life limiting. Patients receive team-based care from a complement of HCPs working on the units. 


\subsection{Project Phases}

The project unfolded in three phases over a six-month period. First, we recruited HCP participants and engaged them in a half-day workshop. The importance of spirituality in person-centred care was discussion and use of the FICA introduced. In the second phase, we conducted an intervention to assess the impact of introducing the FICA as part of patient care on three IP hospital-based units. The third phase focused on data analysis and knowledge translation.

Phase 1: Recruitment and education of HCPS. Recruitment occurred through poster solicitation and invitation by an individual at arms-length from the research team. Three clinical unit managers (one from each site) agreed to facilitate the study. They each designated a member of the IP team to screen clients for inclusion/exclusion criteria, liaise with the Research Assistant (RA) regarding potential study participants, and facilitate access to patient charts. Following recruitment, HCP participants attended a half-day education session during which both the ISCIM and the FICA were introduced. The HCPs had an opportunity to practice taking a spiritual history using the FICA. Written consent to participate in the study was obtained at the beginning of the education session and a pre-education survey was administered to participants. As a follow up to the education, research team members met face to face with HCPs on each of the three units for two focus groups (each 90 min in length) during which HCPs offered preliminary findings related to inclusion of spirituality in patient care and recommendations pertaining to the research protocol.

Phase 2: Data Collection. Patients were screened for eligibility for the study during routine intake, following which the study was described and consent was obtained from those interested. HCP participants conducted a spiritual history during routine patient intake by integrating the FICA into their patient interactions. Findings were documented in patient charts and shared with the IP team. A spiritual care plan was formulated and appropriate referrals were made. Spiritual Care Professionals (SCPs) were available to HCPs for support and guidance. At the end of data collection, HCPs participated in two focus groups: a site-specific focus group and a focus group with all HCPs participants, unit managers and SCPs from the three sites. (See Box 2 for End of Project Focus Group Guiding Questions). Focus groups were audio-recorded and professionally transcribed. Additionally, retrospective chart reviews of study patients were conducted to retrieve all notes regarding HCP inclusion of spirituality in patient care.

Phase 3: Data Analysis. Qualitative data analysis was completed for the focus group transcripts, survey questions, and chart review data. Thematic coding was conducted by three members of the research team (SBP, JW, and RB) using inductive content analysis and NVivo 10 software. To ensure the integrity of the research process, the four criteria of trustworthiness, detailed in Lincoln and Guba's [28] model were addressed throughout the analysis processes. Promoting credibility involved ensuring the study's conceptual description represented the participants' preferences. This study included focus groups in which the HCPs discussed key ideas and reactions to inclusion of spirituality in patient care. Also, research team members cumulatively bring many years of qualitative research, as well as clinical experience and expertise. Maintaining transferability involved using a purposeful and theoretical sampling strategy to identify key informants and satisfy the theoretical needs and comprehensiveness of the conceptual description. Ensuring dependability required examining whether the study process was consistent over time. Over a series of meetings, themes were checked and re-checked, using auditing notes as a source of clarification and to trail decisions made in collapsing 
themes together. Finally, confirmability was ensured through prolonged engagement, including during data collection and analysis, and the extensive use of memos and consultation with co-authors. Techniques of reflexivity and bracketing (researchers' reflection on and articulation of experiences and perceptions related to the research topic) were used to render explicit any idiosyncratic perspectives and potential biases of the researchers. This approach assisted in identifying, hence mitigating bias, to help ensure heightened sensitivity to viewpoints and experiences shared with the researchers.

Box 2. End of project focus group guiding questions.

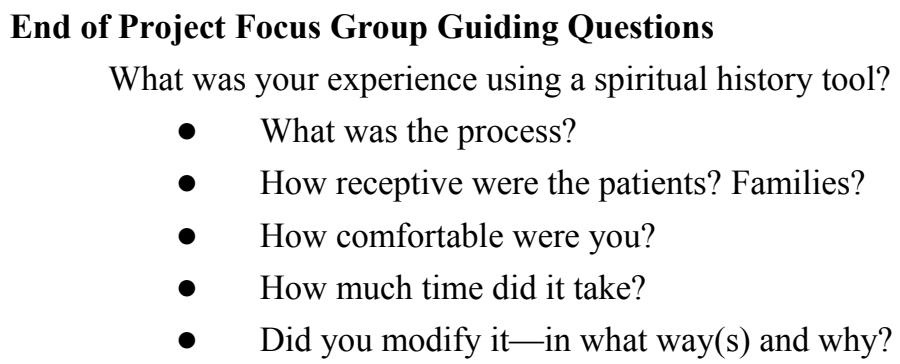

Did the use of a spiritual history tool:

- Facilitate conversation about spiritual issues with:

- Patients or families?

- Colleagues?

- Inform your caregiving? If so, in what ways?

- Did the IP team use the information from the spiritual history tool? How?

Do you think that use of a spiritual history tool would be helpful in assisting other clinicians to integrate spirituality into their clinical practice? Why or why not?

What do you see as potential strengths/barriers of integrating a spiritual history tool:

- Into routine patient care at a clinical/unit level?

- At an organizational level?

What impact, if any, did using a spiritual history tool have on:

- Patient impact and satisfaction?

- You personally and professionally?

- Your clinical practice?

- Your perception of the importance of attending to the spiritual domain?

- Your perception of the role of SCPs?

\section{Results}

\subsection{HCP Participant Characteristics}

Participants recruited for the study across the three units included nine HCPs working on the units (a nurse practitioner, an occupational therapist, a physical therapist, a physician, four registered nurses, and a social worker). HCPs participating in the half-day education session ranged in age from 30 to 59 years and all but one was female. Prior to the session, 11 HCPs (nine of whom actively participated in the project) completed a survey regarding their perspectives on, and comfort with, spirituality in patient care. Survey responses are displayed in Tables 1 and 2. Following the education, HCPs completed a second survey regarding their readiness to administer the FICA and address patient spiritual issues (See responses in Table 3). 
Table 1. Healthcare professional preparation, perspectives, and comfort regarding spirituality in patient care.

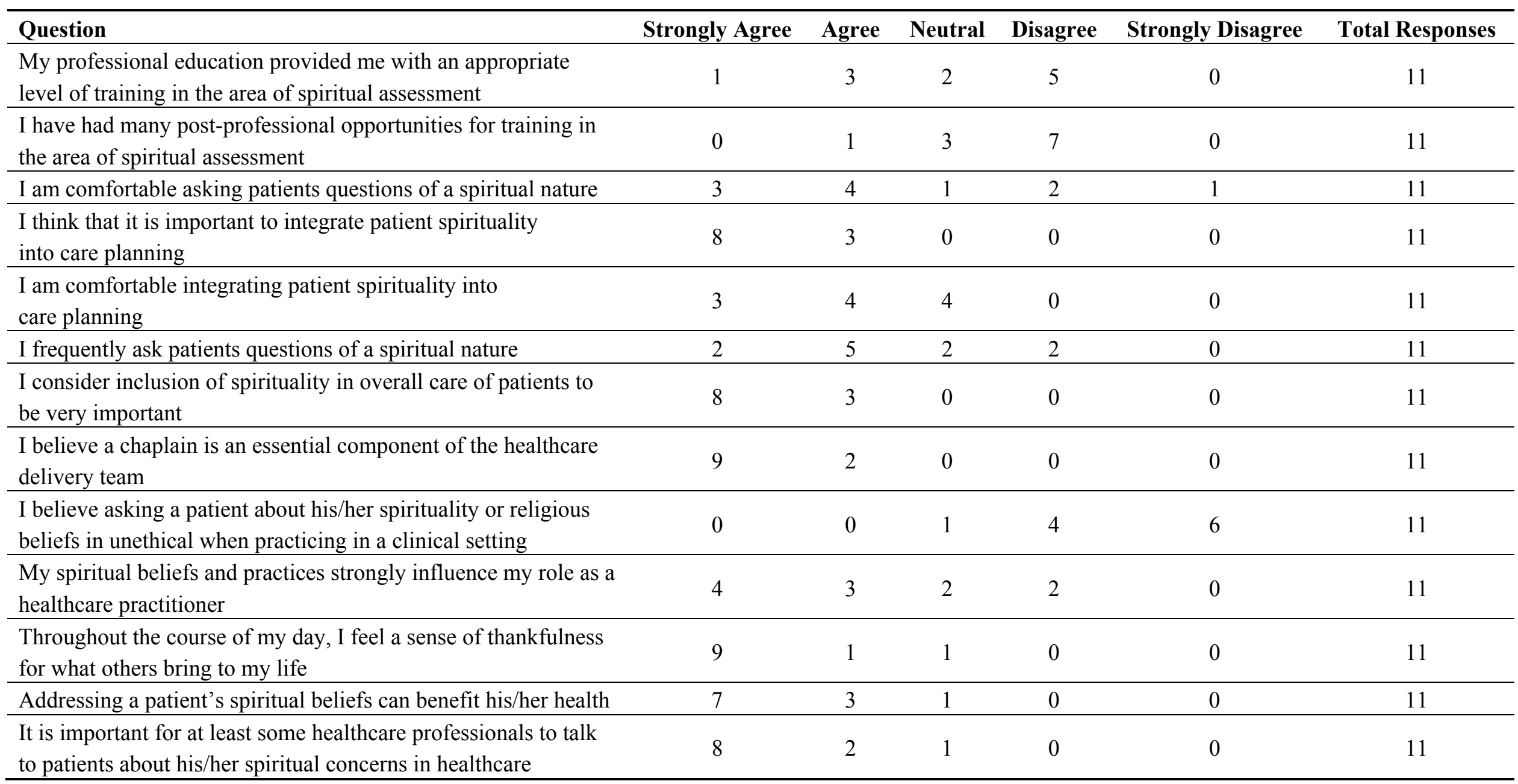


Table 2. Perspectives regarding IP roles \& responsibilities regarding spirituality.

\begin{tabular}{lcccccc}
\hline $\begin{array}{l}\text { I Believe that the Following HCPs are to Discuss } \\
\text { a Patients' Spiritual/Religious Concerns }\end{array}$ & Strongly Agree & Agree & Neutral & Disagree & $\begin{array}{c}\text { Strongly } \\
\text { Disagree }\end{array}$ & $\begin{array}{c}\text { Total } \\
\text { Responses }\end{array}$ \\
\hline Physicians & 3 & 7 & 1 & 0 & 0 & 11 \\
Nurses & 2 & 9 & 0 & 0 & 0 & 11 \\
Physical Therapists & 1 & 8 & 2 & 0 & 0 & 11 \\
Occupational Therapists & 1 & 8 & 2 & 0 & 0 & 11 \\
Social Workers & 2 & 9 & 0 & 0 & 0 & 11 \\
Psychologists & 2 & 8 & 0 & 0 & 1 & 11 \\
Recreational Therapists & 1 & 8 & 2 & 0 & 0 & 11 \\
Music Therapists & 1 & 9 & 1 & 0 & 0 & 11 \\
Pharmacists & 1 & 5 & 3 & 2 & 0 & 11 \\
\hline
\end{tabular}

Table 3. Healthcare professionals preparedness to include spirituality in patient care (post-education).

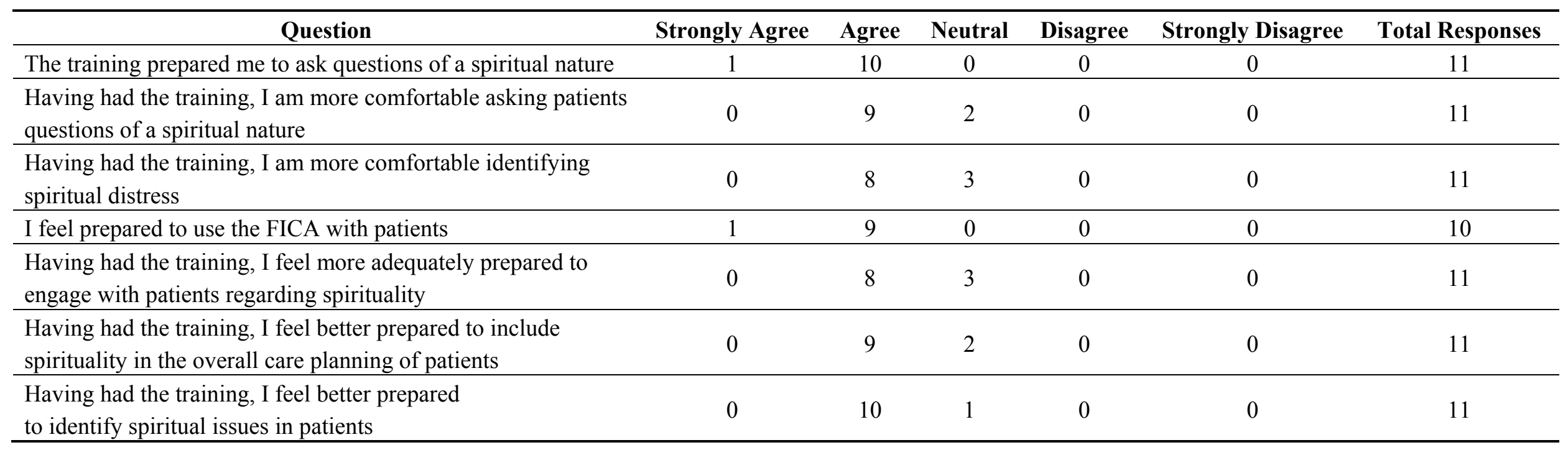




\subsection{Patient Characteristics}

Twenty-four patients were recruited for the study: 10 patients (three male and seven female) ranging in age from 71 to 92 years on the geriatric assessment unit; one 53 year old male on the tertiary palliative care unit; and 13 patients (seven male and six female) ranging in age from 54 to 84 years on the hospice unit. Patients had various diagnoses and symptoms: metastatic cancer $(n=12)$, pain and/or weakness $(n=3)$, dementia $(n=1)$, falls or injury $(n=2)$, gastrointestinal bleed $(n=2)$, COPD $(n=2)$, rehabilitation needs $(n=1)$, and flu-like illness $(n=1)$. Length of hospital stay ranged from 11 to 219 days, while the length of enrolment in the research study was 6-119 days. See Table 4 for a list of patient spiritual issues identified by HCPs and patients, and Table 5 for spiritual interventions offered by the HCPs, and reasons for referral to SCPs.

Table 4. Spiritual issues and interventions.

\begin{tabular}{l}
\hline Spiritual Issues of Patients Identified by Patients and HCPs \\
\hline Need for empowerment, courage, hope, meaning in suffering \\
Grieving, anxiety, lament or protest over loss \\
Sense of being overwhelmed by suffering and uncertain about their ability to endure \\
Difficulty expressing feelings about the situation \\
Expressing guilt, concerns, grief and/or difficulty reflecting on joys, hopes and values \\
Concerns regarding how significant others are coping with illness, loss/changes \\
Coming to acceptance of illness and mortality \\
Fear of dying \\
Abandonment by God and others \\
Religious/spiritual struggles \\
\hline Spiritual Interventions Identified by HCPs to Address Patient Spiritual Issues \\
\hline Referrals to Spiritual Care Professionals \\
Explore issues related to bereavement and loss \\
Active listening, emotional support and emotional expression \\
Allowing sharing of self in discussion, art, music and/or prayer \\
Acknowledgement of the family and its importance in the patient's life \\
Activity and exercise \\
Humour \\
Examination and encouragement of spiritual practices \\
Exploring what is sacred and Divine \\
Spiritual rituals and practices such as prayer, communion, Church attendance \\
Guided visualization, relaxing, breathing
\end{tabular}

Table 5. Interventions offered by IP team members and reasons for referral to SCPs.

\begin{tabular}{ll}
\hline Interventions Provided by HCPs & Reasons for Referral to SCPs \\
\hline Supportive listening & Explore issues related to: \\
Provision of emotional support & Bereavement and loss \\
Being a compassionate presence & Hope/forgiveness and reconciliation \\
Prayer & Meaning of what is sacred and Divine \\
Inclusion of and engagement with family & Examine and encourage religious practice \\
\hline
\end{tabular}


Table 5. Cont.

\begin{tabular}{ll}
\hline Interventions Provided by HCPs & Reasons for Referral to SCPs \\
\hline Facilitation of patient: & Affirm strengths \\
Self-expression through conversation, art, music & Facilitate reception of blessings, rituals and \\
Participation in practices and rituals & sacraments specific to particular faith expressions \\
(e.g., meaningful activities, exercise, Church & \\
attendance, communion, or hymn sing) & \\
\hline
\end{tabular}

\subsection{Key Findings}

The overall purpose of this project was to explore what transpired when HCPs were introduced to a spiritual history tool and given the opportunity to incorporate spiritual history taking into routine patient care. The analysis: (1) explored the value of including spiritual history taking in clinical practice; (2) identified facilitators and barriers to incorporating spirituality into person-centred care; and (3) determined ways in which HCPs can effectively utilize spiritual history taking. The emergent findings include strengths, challenges, and opportunities regarding the inclusion of spirituality in patient care at the organizational, clinical/unit, HCP professional/personal, and patient levels.

\subsubsection{Strengths of HCP Inclusion of Spirituality in Patient Care}

Many strengths related to HCP inclusion of spirituality in patient care were identified. The main strength at the organizational level was the mission, vision, and values of the faith-based institution that served as the study setting. Its guiding documents state that the organization is "committed to serving people of all faiths, beliefs, cultures and circumstances", and caring for the "whole personbody, mind and soul". Another strength was that organizational and clinical leaders, as well as staff, felt aligned with these organizational commitments.

The organization's employment of Spiritual Care Professionals (SCPs) to offer support to patients and staff was identified as a strength at a clinical/unit level. One research participant shared "...our unit actually has spirituality and spiritual assessment very much integrated...so chaplains would be the ones who are doing the spiritual assessment more in-depth and they would get a referral" [29]. SCPs also provide coaching and support to HCPs, thereby cultivating a person-centred culture of care and fostering a "healthy spirituality" on some units.

On a clinical/unit level, staff in some cases were supported by clinical leaders who attended to and role modelled ways of providing care in the spiritual domain. Further, the HCPs functioning on the units were personally and professionally committed to a person-centred approach to care that inherently viewed spirituality as an essential component. Taking a spiritual history supported this approach to care:

It validated why we're here...It makes you more joyous about being able to do your work in this way" [30].

"It's just on my radar even more. So that's really wonderful just to tap into those needs of people" [31]. 
"I think of it as having a conversation, getting to know the patient. If there are any issues, they will get uncovered" [32].

"When we started to incorporate it into our assessment, it just flowed...people talk about community and their family. It was much easier for them to share [29].

HCPs recognized the importance of getting to know, and establishing and maintaining trust with patients and families. One stated, "That person was actually quite happy that we had asked because there were a number of things going on in her life that she really had no one to talk to about. It enabled her to actually ask for help about things that were impeding upon her spiritual state" [33]. Another commented, "It's amazing what people will share with you, their vulnerabilities and everything" [30]. While HCPs were aware of their professional competencies around spirituality, use of a spiritual history tool allowed them to continue to develop, gain confidence with and incorporate it into care. "I see huge value in using a tool and I think this is the core of what we do" [34]. "Just an incredible experience. I'm hoping that this will stay with me. I think it will because I've already had encounters with patients who weren't part of the study with whom I discussed spirituality" [32].

Strengths also emerged at the patient level. Patients and families recognized that spirituality is an essential component of care and they were desirous of having their spiritual issues addressed and integrated into treatment. Inviting patients to share their spiritual perspectives and concerns enhanced their comfort level in the established relationship with the HCP. Patients appreciated that HCPs acknowledged what was meaningful to them and the ways in which this was integrated into their care (e.g., spiritual rituals, liturgy, prayer, art, reflection). Examples included: "A tile named 'A Road Less Travelled' has themes of perseverance, acceptance, not giving up and gratitude." "Intent on completing the tile today, in case something happens to him, at least there will still be the tile" [35]. These spiritual discussions also provided an opportunity for the patient to reflect upon feelings related to their illness and personal experiences. It is also an opportunity for the clinician to diagnose spiritual distress or patient inner resources of strength. Documentation in patient charts indicated, "He struggles with his wish to live and get better and the knowledge that he will not get better", and "It is hard to think of his mortality, finds it scary - is afraid to die" [35]. HCPs were able to find meaning in patient experiences: "Rain drops falling on his face as he smiled stating, "It is a blessing from God. Ahhhhh, this is so good.' As we talked about his faith, the patient thanked the writer for taking him outside" [35]. Discussing what was meaningful to patients also changed how the HCPs interacted with them, enhancing the provision of both spiritual and emotional support. One HCP stated, "I've only done five of them [spiritual screens], but it's amazing how many deep, hurtful things people have to share, because you're listening" [29]. Patients would actively participate in spiritual interventions even at times when they were physically weak and compromised: "Joined in singing hymns and communicating when previously was considered unresponsive" [35].

\subsubsection{Challenges of HCP Inclusion of Spirituality in Patient Care}

HCPs identified a number of challenges at the organizational, clinical/unit, professional/personal, and patient levels when incorporating spirituality into patient care. While using a spiritual history tool was an effective means for eliciting the spiritual issues of patients, participants noted the need to 
develop follow up interventions. Identification of spiritual issues required that time, preparation, and resources were in place to subsequently address them.

A recurrent theme throughout the majority of the interviews was a strong emphasis on patient's physical needs and care. Because patients are often acutely ill, delirious, or cognitively or perceptually compromised, it was difficult to attend to spiritual care needs during hospitalization. Furthermore, there was pressure to discharge or transfer patients prior to spiritual concerns being addressed. Little consideration was given to consistency and continuity of care in this area post-discharge, “...consistency of care...if one unit retains the spiritual information and a patient is transferred, the receiving facility has to start again. In the same way that we work to be consistent with medications during transfers, information about spirituality needs to be shared" [36]. Participants felt that discharge planning was mainly focused on physical issues such as whether the patient was mobile, physically stable, and asymptomatic, "She can walk. She can talk. She can eat. She can drink. Why can't she go home?" [37]. While the mission of the organization clearly supports a whole person focus, the provision of support for spiritual needs seemed to be viewed as extending beyond both essential health care needs and priorities, and the resource capacity of the organization. Overall, fiscal constraints and competing priorities seemed to impede the incorporation of spirituality into patient care. These findings provide rationale for a shift toward the use of the ISCIM [1] in inpatient care environments so as to ensure that spiritual needs are not overlooked.

Recognition of the importance of integrating spirituality into person-centred care requires leadership at the clinical/unit level. Such support makes it more likely that IP team members will address this aspect of care, and that adequate staffing will be in place to meet patient needs. Competing clinical demands - with physical care needs most often taking precedence over spiritual care needscan result in spiritual needs not being addressed, particularly when time is at a premium,

"It's not that we're trying to deny that patient or their family support in that area, it's just that there's a wealth of pressure on each of us to deliver on what we're being asked to do. So we really do need more spiritual care team members in our settings because I don't know that we can all individually take that on" [38].

"We may not have the time or ability to go into the depth of all the religious issues that a patient wants to bring up" [39].

"The barriers were purely logistical and were completely independent of the tool itself which was easy to use" [32].

Even if HCPs were able to find time within their schedules, there was an underlying feeling that using their time to address spiritual needs was not viewed by other IP team members as efficient and productive, “And I just felt like I couldn't justify the time even though I felt it was very important. I felt privileged to be able to be sitting and spending this time with the patient. But I just couldn't relax because I kept thinking I've got to be doing this, this, and this" [40]. At the same time, HCPs noted that taking time to address patients' spiritual needs often facilitated person-centred care.

Communicating patients' spiritual needs to other members of the IP team both verbally and through documentation was noted to be a challenge, "I struggle with the communication of my own plan of care. This is a conversation I've had and how does that then filter through so that the next nurse 
following me or the next team member doesn't have to repeat that whole conversation?" [34]. Without appropriate charting mechanisms or time allocated at a clinical/unit level to communicate findings, issues of inconsistency of approach can arise, "All these sacred moments that I have with patients and with families...how do I capture their essence in the chart? Is that even the place for them? I don't want the moment to be dulled, tarnished, not capturing its beauty in this legal document called a chart" [32].

While addressing spirituality is a core competency, some HCPs felt that they required more knowledge and skills to confidently address spiritual issues, "A lot of information comes out that I'm not trained to deal with and I don't know how to respond to it" [41]. "There's other skills that need to be taught in terms of how to maintain professional boundaries" [39]. HCPs noted a difference between their professional training and that of the SCPs: "She [the SCP] seems to be able to just pile it all on and just keep going about life. And she seems like she's trained and skilled in separating it. These are your issues, this is your path and I'll walk with you down it. But I don't go there" [42]. In the absence of such formation, HCPs may experience more stress/distress, or intentionally or unintentionally cross professional boundaries. Engagement in conversations rather than in a formal process, while potentially indicative of comfort addressing spirituality, also risks diversion away from a patient-centred perspective or imposition of one's personal spiritual views on patients.

The possibility of HCPs experiencing moral/spiritual distress or vicarious suffering was also identified. Moral distress was experienced by HCPs at points when they perceived discord between their morals and values, and the demands placed on them by work, colleagues, families and patients. It also arose when discussing with a patient or family a prognosis, news of impending death, the dying process, or the impact that their illness would have on their quality of life,

"I think I had increased discomfort with this patient's death because of feeling guilt. Did not we, the professionals, hasten her death? By telling her she would move to LTC (long term care)...certainly it appears that the decision made a huge difference. The patient's condition changed drastically. After that she essentially gave up" [32].

Patient understandings of spirituality and their ability to speak about it impacted both their level of engagement in this area and the degree to which spirituality was integrated into their care. Patients who did not consider themselves to be "spiritual" refrained both from engaging in this area and involvement in the study, "(Patient) did not want to be part of 'anything big', and requested to be left alone" [43]. "I mentioned the research project and asked if I could talk with him. I got the same response again: 'Oh, I am not very spiritual, I do not know why I signed up for this"' [35]. Other patients were very private regarding their spirituality, "(She) said her spirituality was only her business and didn't wish to share" [43]. Some patients also became defensive when spirituality was raised, "I started to explain that spirituality is whatever is important to him. He looked at me and said, 'To hell with it, I'm out" [32]. Language barriers further limited engagement with some patients, "I did find a challenge with one woman who didn't speak very much English. I couldn't get words out of her at all' [31]. Patient engagement around spiritual issues at times resulted in patients having a false perception of connectedness with and attachment to an HCP, “I had one negative experience where I didn't spend enough time with an individual after conducting the spiritual history. They felt I didn't love them anymore and that's what they told me, and so it actually turned kind of negative, which was 
unfortunate" [29]. The discussions brought a sense of closeness. When the HCP did not continue to engage in the same manner, the patient experienced distress.

A final challenge of note related to continuity of spiritual care upon transition to another clinical unit or discharge into the community. Spiritual resources were not consistently available at the time of transition and HCPs did not know how to access them, "I don't know what a treatment plan is for referring on for community follow up regarding spirituality" [39]. Further, the relationship with the HCP(s) who had engaged a patient on a spiritual level ended, leaving some patients feeling devastated, ambiguous and unsure of the future,

"They gain a trust with the team, they feel comfortable, they gain that sense that I'm going to be alright, this is going to be OK, I am at the end of my journey, I'm safe. And then when we throw in that mix 'no, you've stabilized and you no longer need us', their world is turned upside down. Patients feel rejected. Often we see patients will just give up" [44].

HCP engagement in the spiritual domain is not without its challenges.

\subsubsection{Opportunities for HCP Inclusion of Spirituality in Patient Care}

There were many opportunities related to inclusion of spirituality in patient care at the organizational, clinical/unit, professional/personal, and patient levels. The funding of this research study - provided by an organization to explore ways to more explicitly include spirituality in patient care - is one example of an opportunity seized upon by an organization. Research days supported by this organization that are specifically focused on themes such as spirituality in healthcare, provide vehicles for dissemination of research findings and examination of clinical best practices. Further, the organization's openness to ongoing work with researchers on this topic, as well as financial and professional support to staff who decide to further their learning in this area, are additional examples of an approach that can be used to align an organizational mission with practice.

At the clinical/unit level, a more systematic and integrated approach to inclusion of spirituality in patient care may enhance patient and family satisfaction. Patients and families may perceive that the IP team is more attentive to and respectful of the patient as a person rather than as a constellation of physical symptoms. As well, satisfaction among HCPs could increase as their awareness of the central role of spirituality in the provision of healthcare increases, "It's a positive component. Being able to be part of the project and using this tool is just great. There's a framework which helps my confidence with patients, and gives me greater awareness again" [45]. Some HCPs were of the opinion that spirituality at the clinical/unit level would be more likely and consistently addressed if a specific tool were incorporated on an ongoing basis. HCPs felt that this would facilitate patient engagement around spiritual matters within the context of routine care, such as while walking with a patient, or during a therapy sessions. Some HCPs also questioned whether patients, on return admissions, would continue to want to address their spiritual concerns, "I think what's piqued my curiosity is when these patients that took part in the study come back (because they always do), will they be more willing to talk about their spiritual needs?" [46].

At the professional/personal level, HCPs have many opportunities to more intentionally include spirituality in patient care and become comfortable addressing this area, 
"I found that at first administering the tool was very awkward. It was just very rehearsedbringing the tool in with me, going over it with the patient. But then you develop a game plan and you learn what to listen for" [36].

"As time progresses, you hear those keywords that the patients are saying and you can pick up on that because you're more familiar with the tool" [36].

"I think this allowed other components to be looked at. I think it really brought some enlightenment to some of the team, it opened up questions, and dialogue" [29].

An opportunity for increased job satisfaction was identified, with HCPs being better able to meet patient needs, "So it always is helpful to go deep because then you find out more and you get to know the person. You could work with them that way" [47]. As well, attending to the spiritual component of patient care validated the HCPs' professional role/vocation, "I think I boiled it down to this connection with people. So even if I couldn't have a conversation with someone because they were too ill, just the spark in their eyes, there's a connection. I'm connecting with this person and this person is connecting with me and we both know it, we can just feel it in the air. And I think I've had that before, but going through, doing the whole spiritual history, it just became so very present in my mind again. It wasn't at the back in my mind, it was at the forefront again" [48]. "I think it gives us an identity too. Because traditionally this institution is faith-based. But what do people see when they walk through the door that distinguishes us? So I think asking these questions kind of tells patients that we are different. We're actually interested in your spiritual needs at this time...during your moment of crisis when being admitted to hospital" [46].

Addressing spiritual issues also affords HCPs opportunities for professional growth. Some HCPs were better able to establish therapeutic relationships,

"I think my interactions with patients and families has just been so enriched...just getting to that connection, that core...I see the patient as a human being, not just as a frail, ill cancer patient. I see a person and a soul, the essence of them shining through, connecting on that level" [48].

Other HCPs were able to improve their communication and empathic capacity,

"I think we're here to try to provide care and comfort and quality to the remaining days that an individual has. So, it adds that ability because we're looking at that individual person and asking what matters to them...it could be as simple as taking that individual up to the roof top for fresh air" [45].

Finally, there is an opportunity for greater IP collaboration. Inclusion of spirituality provides common ground upon which all members of the IP team can collaborate. Some HCPs seemed surprised to learn that other disciplines have the ability to address the spiritual dimension,

"As the nurses were reading the charts, especially the spiritual histories that got put into the charts, a lot of them commented to me, 'Oh, I never really thought of an occupational therapist addressing spirituality, or a physiotherapist addressing spirituality as part of their role. So I think that was kind of an eye opener'" [49]. 
This finding presents a rich opportunity for IP learning and collaboration.

Attending to patient needs inclusive of spirituality presents opportunities to enhance the patient experience of care. Patients, families and HCPs recognized an overall increase in satisfaction when spiritual concerns were integrated as part of patient care planning. When patients were invited to share what they consider to be personally meaningful to them, HCP's were able to incorporate these components into their care plan,

"He was young. He just said 'I just want to feel the sunshine on my face one more time'. So somebody listened. It was five minutes. But what a beautiful bag to open instead of 'It's not medical so it doesn't fit within my framework or my context or my priorities"” [50].

HCP's also indicated that there have been fewer call bells heard on the unit since use of the FICA was introduced, and believed that the quality of care offered to patients was enhanced,

"In my mind, there is improved quality, care and comfort for our patients" [29].

"Spirituality should be practical and visible. He sees staff caring for him as representation of this" [35].

Participants felt that an opportunity existed for inviting family members to share their perspectives, and believed that there could be additional value in having both the patient and their family involved in completing a spiritual history. HCPs noted that families have good insight into a patient's spiritual values and concerns, and felt that they could assist in facilitating better spiritual care for the patient,

"I find I spend more time with the family than with the patient because it's the families that seem to have more of the questions. The patients seem to be, well, some of them, are not able to speak. So it's the families that I feel I'm more valuable to regarding the whole connection thing" [40].

The discussions provided an opportunity for patients to find meaning, grow, express themselves, and be empowered. As an example,

"Had image on a tile: a rock with a flower growing out of it. Patient found it awkward to share what symbol/image means to him. More comfortable in speaking of what it means to people. Words used were renewal and beginning of a cycle. Resonated with the idea that people have unique perspectives on the world and there is value in sharing it" [35].

Engaging in spiritual discussions can enhance the patient's experience, foster connectedness, and promote opportunities for further spiritual growth.

Patients reportedly appreciated being treated with dignity and respect, and connecting with what is most meaningful to them,

"I have worked on quite a lot of the units in the hospital and so I really do see a value, especially with patients that are facing end of life issues, who are dealing with significant health issues that are changing their abilities to manage. It is a valuable way to look at coping skills, your support systems, and how you draw on your faith to strengthen you to get through some of these moments" [51]. 
Having an awareness of what is meaningful to a patient enables HCPs to better help patients cope with their current and future circumstance,

"It was stormy out so the patient suggested that writer take him to another area of interest, looking at art. He was interested in color and texture. He spoke of his image of the flower in the rock on the tile. Meaning to him was a hopeful image of strength, surprise and gratitude" [35].

Discussions such as this can help a patient draw on spiritual resources in a time of crisis.

The inclusion of spirituality in care allowed for both the identification and reduction of existential suffering, and an opportunity to support patients in addressing unresolved spiritual issues, "The patient is so willing to share his feelings, his vulnerabilities. What a gift!" [32]. With each subsequent meeting, the $\mathrm{HCP} /$ patient relationship developed further and spiritual sharing continued, "Told HCP she wanted to die and that she has had enough. Things are changing and she is at peace. She spoke of her feelings about dying" [35]. Often referrals to SCP's were made as concerns were identified by the HCP's, which provided patients with additional spiritual and emotional support as they journeyed through their illness. Patients shared feeling at peace as they faced their illness and future passing, "Patient's reflections on death are very cathartic and thoughtful" [35].

The opportunity to explore additional spiritual resources, especially those that are community-based, arises when patients transition to another clinical unit or are discharged from hospital-based care. So as to ensure continuity of care, spiritual information gathered in one setting needs to be integrated into the next, "I think it helps as people near the end of life that they can actually have all those little pieces put together. It helps with advanced planning and future directions" [52].

\section{Discussion}

This study examined the effect of integrating spirituality into patient care within in-patient care settings by IP teams. Specifically, this project was undertaken to: (1) explore the value of including spiritual history taking in clinical practice; (2) identify facilitators and barriers to incorporating spirituality into person-centred care; and (3) determine ways in which HCPs can effectively utilize spiritual history taking. It yielded initial findings to support proposals for future collaborative multisite intervention studies. Overall, findings from this study contribute to a growing body of knowledge and evidence supporting the inclusion of spirituality in person-centred care by IP team members. HCPs indicated that the study facilitated a greater awareness of the need for all HCPs to possess the ability to recognize and respond to spiritual needs whenever and wherever they might arise. They also acknowledged ways in which attention to spirituality facilitated connection with patients, improved person-centred care, and enhanced their sense of job satisfaction when meeting patient needs. These findings suggest that, in addition to positive outcomes associated with improved patient care, attending to spiritual concerns may potentially also help to reduce rates of burnout among IP team members. Further, the study offered new insights into requirements that would support more intentional inclusion of the spiritual component in patient care at a variety of levels, including organizational, clinical/unit, professional/personal, and patient levels. 


\subsection{Incorporation of Spirituality}

Incorporation of spirituality at a unit level was accomplished by integrating the tool into routine professional practice. This was found to be helpful in initiating and guiding spiritual conversations. More education and greater support for HCPs is required so that they can more competently, confidently and intentionally attend to the spiritual dimension of patient care. This education might include opportunities to (1) enhance self-awareness; (2) improve the ability to differentiate patient distress from one's own spiritual discomfort; (3) develop competencies and skills in spiritual history taking, interviewing and interventions; (4) explore ways to adhere to professional boundaries; and (5) practice responding to and reflecting on spiritual issues using a team-based, collaborative approach consistent with the ISCIM. Such education would be beneficial in undergraduate and graduate level curricula, and continuing education course offerings.

Facilitators (strengths) and challenges related to the incorporation of spirituality in person-centred care were explored. Findings suggest that, while inclusion of spirituality can be inherent in an organization's mission and vision, competing priorities, increasing fiscal constraints, urgency to discharge patients, and limited resources can compromise attention given to this area of patient care. There is potential for greater impact if the organization's mission, vision and values are followed up by explicit initiatives to help to advance the mission. These could include pilot studies, ongoing professional education offerings, supportive communication including introduction of change, as well as change management. HCPs identified a challenge regarding documentation following inclusion of spiritual history taking and intervention. Such an issue would best be addressed at the organizational and clinical/unit levels, as well as in education around spiritual history taking and intervention.

\subsection{Study Limitations and Lessons}

Limitations of the study include (1) gaps in HCP knowledge regarding inclusion of spirituality in patient care; (2) the small number of HCP and patient study participants; (3) participants being drawn exclusively from a single faith based healthcare organization; (4) brevity of the training sessions for HCPs; (5) limited follow-up with HCP participants regarding integrity of use of the FICA tool (e.g., which questions were asked and how the tool was used); and (6) challenges with the consenting process when working with vulnerable patients. Barriers to recruitment of HCPs were related to competing priorities and self-perceived inappropriateness for this type of study due to their limited understanding of, and confidence in, the area of spirituality. Some patients were reluctant to participate due to their perceptions of spirituality, while others did not consider themselves as "spiritual", and therefore did not see themselves as appropriate for the study. Declining health further precluded patient involvement in the study, as did unfortunate timing of engagement with patients around spiritual issues. Clients also often required immediate engagement in the area of spirituality once the conversation was initiated. Delays in provision of spiritual history taking and support at times resulted in patients not being open to engage further in this area. While the faith-based setting was considered to be a strength in that it facilitated and provided spiritual support from certified SCPs and other HCPs, results may vary in other institutions, including secular healthcare organizations that have differing missions, visions, and values. 
Based on the experience of the research team and the findings of this study, we have learned that careful planning, collaborative consultations, and responsive adjustments are required when conducting research with HCPs caring for vulnerable populations. Further research in this area would require: (1) more extensive education for HCPs around use of the spiritual history tool; (2) evaluation of HCP fidelity to assessment protocol when using the spiritual history tool with various vulnerable populations; (3) larger numbers of participants; and (4) a longer trial period for HCP engagement and follow up with patients; Furthermore, we recommend (5) engagement with diverse IP teams working with various patient populations; and (6) inclusion of settings that are guided by both faith- and non-faith-based missions. Additional contributions to the growing body of knowledge in this area could be made through such research, thereby providing further evidence to support inclusion of spirituality in the provision of patient care.

\section{Conclusions}

This paper has highlighted research study findings related to the integration of spirituality in patient care within in-patient care settings by interprofessional teams. Such integration was found to enhance person-centred care, foster connection with patients, improve HCP job satisfaction and reduce burnout among IP team members. It also facilitated awareness of the need for recognition of and a response to patient spiritual needs in a timely manner. More intentional inclusion of the spiritual component in patient care at organizational, clinical/unit, professional/personal, and patient levels might be better supported through the mission and vision of an organization, support from leadership, provision of education and support for HCPs in the development of competencies and confidence addressing the spiritual domain, and consistent use of spiritual history tools in routine practice.

While inclusion of spirituality in patient care was found to have a significant impact on both patients and HCPs alike, competing priorities, fiscal constraints, urgency to discharge, documentation-related challenges and limited resources risk compromising attention given to this important area of patient care. Such a reduction has the potential to negatively effect patient care offered by IP teams, and the ability of HCPs to both relate to patients as people, and effectively help those reliant on them for care. Addressing the spiritual domain of individuals in care clearly has a positive influence on patient care, IP team members, and overall organizational culture. Its inclusion is therefore important when considering ways to effectively deliver high quality healthcare services.

\section{Acknowledgments}

The authors thank Roopa Belur (Research Assistant) and Brent Watts (Chaplain Specialist in Spiritual Care with Alberta Health Services/University of Alberta Hospital) for their contributions to the data analysis and formulations of recommendations for this project. We thank Covenant Health for funding this project, and the HCPs and patients who made this study possible through their participation. Covenant Health is committed to its faith-based vision and mission, a person-centred care approach, interprofessional teamwork, and research, all of which were critical to this project. Their willingness and desire to open their doors to researchers provides an opportunity to examine effective ways in which spirituality can be included in patient care. 


\section{Author Contributions}

All authors substantively contributed to the research project that informed the findings reported in the article. Suzette Brémault-Phillips was the P.I. of the study. She along with Joanne Olson, Pamela Brett-MacLean, Doreen Oneschuk, Shane Sinclair, Ralph Magnus, Marjan Abbasi, Jasneet Parmar, and Christina M. Puchalski developed the proposal for funding. Christina M. Puchalski developed the spiritual history tool utilized in this project, and provided permission for its use. Suzette Brémault-Phillips, Joanne Olson and Shane Sinclair wrote the application for ethical approval of the study. Suzette Brémault-Phillips, Joanne Olson, Shane Sinclair, Ralph Magnus and Christina M. Puchalski participated in the education day. Suzette Brémault-Phillips, Joanne Olson, Pamela Brett-MacLean, Doreen Oneschuk, Shane Sinclair, Ralph Magnus, Jasneet Parmar, and Christina M. Puchalski participated in the research project meeting that followed. Mid-project follow-up focus groups with HCP participants were conducted by Suzette Brémault-Phillips, Ralph Magnus, and Jeanne Weis. Marjan Abbasi attended one follow-up focus group. Final focus groups were conducted by Suzette Brémault-Phillips, Pamela Brett-MacLean, Doreen Oneschuk, Ralph Magnus, and Jeanne Weis. Jasneet Parmar attended a team meeting that followed. Patient consenting was done by Jeanne Weis and Suzette Brémault-Phillips, and chart reviews were done by Jeanne Weis. Suzette Brémault-Phillips, Jeanne Weis, and Joanne Olson were involved in data analysis. The manuscript was drafted by Suzette Brémault-Phillips, Joanne Olson, Pamela Brett-MacLean, Shane Sinclair, Doreen Oneschuk, and Jeanne Weis. All team members contributed to revising the final manuscript and provided their approval for authorship.

\section{Abbreviations}

HCP: Healthcare Professional;

IP: Interprofessional;

FICA: FICA Spiritual History Tool@;

RA: Research Assistant;

RN: Registered Nurse;

SCP: Spiritual Care Professional.

\section{Conflicts of Interest}

Ralph Magnus is an employee of the funding organization, while Doreen Oneschuk, Marjan Abbasi, and Jasneet Parmar conduct clinical practice within the funding organization. Christina M. Puchalski developed the spiritual history tool utilized in this project.

\section{References and Notes}

1. Christina M. Puchalski, Betty Ferrell, Rose Virani, Shirley Otis-Green, Pamela Baird, Janet Bull, Harvey Chochinov, George Handzo, Holly Nelson-Becker, Maryjo Prince-Paul, and et al. "Improving the quality of spiritual care as a dimension of palliative care: The report of the consensus conference.” Journal of Palliative Medicine 12 (2009): 885-904. 
2. Christina M. Puchalski. "Spirituality and health: The art of compassionate medicine." Hospital Physician 37 (2001): 30-36.

3. Mark Cobb, Christopher Dowrick, and Mari Lloyd-Williams. "What can we learn about the spiritual needs of palliative care patients from the research literature?" Journal of Pain and Symptom Management 43 (2012): 1105-19.

4. Karen E. Steinhauser, Nicholas A. Christakis, Elizabeth C. Lipp, Maya McNeilly, Lauren McIntyre, and James A. Tulsky. "Factors considered important at the end of life by patients, family, physicians, and other care providers.” The Journals of American Medical Association 284 (2000): 2476-82.

5. Daren Heyland, Peter Dodek, Graeme Rocker, Dianne Groll, Amiram Gafni, Deb Pichora, Sam Shortt, Joan Tranmer, Neil Lazar, Jim Kutsogiannis, and Miu Lam. "What matters most to patients." Canadian Medical Association Journal 174 (2006): 627-33.

6. Shane Sinclair, Jose Pereira, and Shelley Raffin. "A thematic review of the spirituality literature in palliative care." Journals Palliative Medicine 9 (2006): 464-79.

7. Moira Stewart, Judith Belle Brown, Allan Donner, Ian R. McWhinney, Julian Oates, Wayne W. Weston, and John Jordan. "The impact of patient-centred care on outcomes." Family Practice 49 (2000): 796-804.

8. Moira Stewart, Bridget L. Ryan, and Christina Bodea. "Is patient-centred care associated with lower diagnostic costs?" Healthcare Policy 6 (2011): 27-31.

9. Tracy A. Balboni, Lauren C. Vanderwerker, Susan D. Block, M. Elizabeth Paulk, Christopher S. Lathan, John R. Peteet, and Holly G. Prigerson. "Religiousness and spiritual support among advanced cancer patients and associations with end-of-life treatment preferences and quality of life." Journal of Clinical Oncology 25 (2007): 555-60.

10. Harvey Max Chochinov, Thomas Hassard, Susan McClement, Thomas Hack, Linda J. Kristjanson, Mike Harlos, Shane Sinclair, and Alison Murray. "The landscape of distress in the terminally ill." Journal of Pain and Symptom Management 38 (2009): 641-49.

11. Christina M. Puchalski, Robert Vitillo, Sharon K. Hull, and Nancy Reller. "Improving the spiritual dimension of whole person care: Reaching national and international consensus." Journal of Palliative Medicine 17 (2014): 642-56.

12. Shane Sinclair, and Harvey Max Chochinov. "Communicating with patients about existential and spiritual issues: SACR-D work.” Progress in Palliative Care 20 (2012): 72-78.

13. Christina M. Puchalski. "The role of spirituality in health care." Proceedings (Baylor University Medical Center) 14 (2001): 352-57.

14. John W. Ehman, Barbara B. Ott, Thomas H. Short, Ralph C. Ciampa, and John Hansen-Flaschen. "Do patients want physicians to inquire about their spiritual or religious beliefs if they become gravely ill?” JAMA Internal Medicine 159 (1999): 1803-06.

15. Shane Sinclair, Shelley Raffin Bouchal, Harvey Chochinov, Neil Hagen, and Susan McClement. "Spiritual care: How to do it." BMJ Supportive and Palliative Care 2 (2012): 319-27.

16. Gallup International Institute. Spiritual Beliefs and the Dying Process: A Report on a National Survey. Princeton: Gallup International Institute, 1997. 
17. David Hui, Maxine de la Cruz, Steve Thorney, Henrique A. Parsons, Marvin Delgado-Guay, and Eduardo Bruera. "The frequency and correlates of spiritual distress among patients with advanced cancer admitted to an acute palliative care unit." American Journal of Hospice \& Palliative Medicine 28 (2011): 264-70.

18. Caterina Mako, Kathleen Galek, and Shannon Poppito. "Spiritual pain among patients with advanced cancer in palliative care." Journal of Palliative Medicine 9 (2006): 1106-13.

19. Lucy Selman, Teresa Young, Mieke Vermandere, Ian Stirling, and Carlo Leget. "Research priorities in spiritual care: An international survey of palliative care researchers and clinicians." Journal of Pain and Symptom Management 48 (2014): 518-31.

20. Mark Ellis, James D. Campbell, Ann Detwiler-Breindenbach, and Dena K. Hubbard. "What do family physicians think about spirituality in clinical practice?" Journal of Family Practice 51 (2002): 249-54.

21. Anita Molzahn, and Laurene Shields. "Why is it so hard to talk about spirituality?" Canadian Nurse 104 (2008): 25-29.

22. Christina M. Puchalski, Beverly Lunsford, Mary H. Harris, and Rabbi Tamara Miller. "Interdisciplinary spiritual care for seriously ill and dying patients: A collaborative model." The Cancer Journal 12 (2006): 398-416.

23. Shane Sinclair. "Impact of Death and Dying on the Personal and Professional Lives of Palliative Care Professionals.” Canadian Medical Association Journal 183 (2011): 180-87.

24. FICA Spiritual History Tool, (C) C. Puchalski, 1996. Please contact Dr. Puchalski for permission to use the FICA tool at cpuchals@gwu.edu.

25. Christina M. Puchalski, and Anna L. Romer. "Taking a spiritual history allows clinicians to understand patients more fully." Journal of Palliative Medicine 3 (2000): 129-37.

26. Christina M. Puchalski. "The FICA Spiritual History Tool \#274." Journal of Palliative Medicine 17 (2014): 105-06.

27. Tami Borneman, Betty Ferrell, and Christina M. Puchalski. "Evaluation of the FICA Tool for Spiritual Assessment.” Journal of Pain and Symptom Management 40 (2010): 163-73.

28. Yvonna S. Lincoln, and Egon G. Guba. Naturalistic Inquiry. Beverly Hills: Sage, 1985.

29. Interviewee S-FG-b (Covenant Health, Edmonton, Alberta). Interview, 2014.

30. Interviewee S-FG-A-4 (Covenant Health, Edmonton, Alberta). Interview, 2014.

31. Interviewee JN-FG-A-2 (Covenant Health, Edmonton, Alberta). Interview, 2014.

32. Interviewee S-FG-E (Covenant Health, Edmonton, Alberta). Interview, 2014.

33. Interviewee S-FG-B-2 (Covenant Health, Edmonton, Alberta). Interview, 2014.

34. Interviewee JN-FG-A-3 (Covenant Health, Edmonton, Alberta). Interview, 2014.

35. Interviewee (Covenant Health, Edmonton, Alberta). Chart Documentation, 2014.

36. Interviewee S-FG-A-3 (Covenant Health, Edmonton, Alberta). Interview, 2014.

37. Interviewee J-FG-b-2 (Covenant Health, Edmonton, Alberta). Interview, 2014.

38. Interviewee S-FG-B-3 (Covenant Health, Edmonton, Alberta). Interview, 2014.

39. Interviewee JL-FG-B-2 (Covenant Health, Edmonton, Alberta). Interview, 2014.

40. Interviewee JN-FG-A-5 (Covenant Health, Edmonton, Alberta). Interview, 2014.

41. Interviewee S-FG-B-1 (Covenant Health, Edmonton, Alberta). Interview, 2014. 
42. Interviewee JL-FG-b-2 (Covenant Health, Edmonton, Alberta). Interview, 2014.

43. Interviewee (Covenant Health, Edmonton, Alberta). Patient Declines Note, 2014.

44. Interviewee S-FG-B (Covenant Health, Edmonton, Alberta). Interview, 2014.

45. Interviewee JL-FG-A-3 (Covenant Health, Edmonton, Alberta). Interview, 2014.

46. Interviewee JL-FG-B-1 (Covenant Health, Edmonton, Alberta). Interview, 2014.

47. Interviewee JL-FG-B-3 (Covenant Health, Edmonton, Alberta). Interview, 2014.

48. Interviewee S-FG-b-3 (Covenant Health, Edmonton, Alberta). Interview, 2014.

49. Interviewee S-FG-B-4 (Covenant Health, Edmonton, Alberta). Interview, 2014.

50. Interviewee JL-FG-A-1 (Covenant Health, Edmonton, Alberta). Interview, 2014.

51. Interviewee S-FG-b-2 (Covenant Health, Edmonton, Alberta). Interview, 2014.

52. Interviewee (Covenant Health, Edmonton, Alberta). Reflection 7, 2014.

(C) 2015 by the authors; licensee MDPI, Basel, Switzerland. This article is an open access article distributed under the terms and conditions of the Creative Commons Attribution license (http://creativecommons.org/licenses/by/4.0/) 